\title{
Editorial: Cell Biology, Physiology and Molecular Pharmacology of G Protein Coupled Receptors
}

\author{
Manveen Kaur Gupta ${ }^{1}$, Muheeb Beg ${ }^{2}$ and Sameer Mohammad ${ }^{3 *}$ \\ ${ }^{1}$ Cardiovascular and Metabolic Sciences, Lerner Research Institute, Cleveland Clinic \& Case Western Reserve University, \\ Cleveland, OHIO, United States, ${ }^{2}$ Department of Medical Biochemistry and Cell Biology, Institute of Biomedicine, Sahlgrenska \\ Academy, University of Gothenburg, Gothenburg, Sweden, ${ }^{3}$ Experimental Medicine, King Abdullah International Medical \\ Research Center (KAIMRC), King Saud Bin Abdulaziz University for Health Sciences (KSAU-HS), Ministry of National Guard Health \\ Affairs (NGHA), Riyadh, Saudi Arabia
}

Keywords: GPCR (G protein coupled receptor), adrenergic receptor (AR), GPCR drug discovery, GRK (G protein receptor kinase), Incretin - Based therapy

\section{Editorial on the Research Topic}

Cell Biology, Physiology and Molecular Pharmacology of G protein Coupled Receptors

G protein-coupled receptors (GPCRs) are the largest family of cell-surface proteins. They are characterized by seven transmembrane domains; a cytoplasmic C-terminus, and an extracellular N-terminal domain. GPCRs respond to a wide variety of signaling molecules and signal through the trimeric G-protein complex. GPCRs are expressed essentially on all cells, facilitating cellular responses to external stimuli and are involved in nearly every biological process (Pierce et al., 2002; Strange, 2008; Hanyaloglu and Grammatopoulos, 2017; Pavlos and Friedman, 2017; Wang et al., 2018). There are $~ 800$ members of the GPCR family, of them more than 400 are sensory receptors (olfactory, vision, and taste receptors (Alexander et al., 2019). The remaining $\sim 350$ are non-sensory receptors and are activated by physical ligands, which include peptide hormones, large polypeptides, amino acids and small metabolites, free fatty acids and many others (Okuno et al., 2006; Mohammad, 2015; Wolf and Grünewald, 2015; Husted et al., 2017; Al Mahri et al., 2020; Davenport et al., 2020). Based on sequence homology, GPCRs are divided into different families: Class A (rhodopsin), Class B (secretin, adhesion), Class C (Glutamate), and frizzled receptors (Alexander et al., 2019). Most of these receptors have a known physical ligand, which activates the receptor to elicit the signaling cascade and the downstream effect on physiological function. GPCRs are substantially involved in human pathophysiology and are pharmacologically tractable, making them the most intensely studied drug targets. Nearly one-third of all drugs approved by the US Food and Drug administration involve GPCR target sites. In addition, several GPCR based drugs are currently undergoing clinical trials (Nieto Gutierrez and McDonald, 2018; Sriram and Insel, 2018). However, only about 100 GPCRs have been extensively studied and successfully targeted while the functional relevance of a significant number of GPCRs remains to be studied. Therefore, intense efforts are on to expansively study the rest of the members of the GPCR family to unearth additional therapeutic possibilities.

This research topic comprises of research papers and review articles on diverse GPCRs and their regulators. The collection of articles highlights the role of GPCRs in physiology, pathophysiology, and explores the possibility of exploiting their therapeutic potential. Perez (2021) reviews current developments on the role of $\alpha 1$-Adrenergic Receptors ( $\alpha 1$-ARs) in cognition, cardioprotection, and metabolism. $\alpha 1$-ARs belong to the family of adrenergic receptors and have been extensively studied

Front Cell Dev. Biol. 9:815291. doi: $10.3389 /$ fcell.2021.815291 for their role in blood pressure regulation, cardiac hypertrophy, and muscle contraction. $\alpha 1$-ARs are 
also highly expressed in the cognitive centers of the brain and their activation has a profound effect on learning and memory function. The review highlights the potential of $\alpha 1 \mathrm{~A}-\mathrm{AR}$ agonists or positive allosteric modulators to treat Alzheimer's disease and to protect the heart at the same time.

Lin et al. (2021) in their paper demonstrate the role of Adenosine Receptor (AdoR) signaling and its downstream targets in Drosophila. They demonstrate that AdoR signaling represents an important pathway in response to stress conditions such as cytotoxicity, oxidative damage, and thermal stress in Drosophila. The paper provides important insights into the molecular mechanism of Ado regulation of stress response that could help understand how Ado signaling affects disease pathogenesis.

An interesting paper by Chou et al. (2021) shows how G-Protein-Coupled Estrogen Receptor-1 (GPER-1) positively regulates chondrocyte proliferation at the growth plate during early puberty and contributes to the longitudinal growth of long bones. GPER-1 is widely expressed in both mouse and human tissues including bone and cartilage. Interestingly, during pubertal progression, the expression of GPER-1 shows a significant decrease suggesting its involvement in the modulation of pubertal bone growth. Previous studies have investigated the expression of GPER-1 in bone and cartilage but the functional significance of GPER-1 in bone growth remains unclear. Using chondrocyte-specific GPER-1 knockdown mice, the authors demonstrate that GPER-1 positively regulates chondrocyte proliferation at the growth plate during early puberty and contributes to the longitudinal growth of long bones.

In a comprehensive review (Morrow et al., 2021), critically assess the role of incretin receptors, GIP receptor (GIPR), GLP-1 receptor (GLP-1R), and GLP-2 receptor (GLP-2R) in intestinal physiology. Incretin hormones (GIP and GLP-1) are gut peptides that are released from intestinal L- and K-cells respectively in response to food intake. Once released into the bloodstream, they bind to incretin receptors (GIPR. GLP-1R and GLP-2R) in pancreatic $\beta$-cells and enhance insulin releases in a glucosedependent manner. In subjects with type 2 diabetes, this incretin effect is diminished or no longer present. Therefore, incretin-based therapies have been successfully used in diabetic patients. The authors in this paper highlight the biology and paracrine roles of GLP-1, GIP, and GLP-2 in integrating the response to food intake with the maintenance of the structure and function of the gut as it relates to nutrient absorption. A thoughtprovoking paper included in this research topic is by Kizilkaya et al. (2021) on incretin receptor GIPR. The authors functionally characterized two missense GIPR variants, R190Q (rs139215588) and E288G (rs143430880) that are associated with lower body mass index (BMI). The authors show that two naturally occurring rare GIPR variants, R190Q and E288G (rs139215588 and rs143430880, respectively), result in impaired GIPR function at the molecular level which in turn seems to impact human physiology and pathophysiology regarding adiposity, bone health, and cardiovascular system. These results indicate that GIPR antagonists could protect from diet-induced obesity and improve glycemic and insulinotropic effects, which is in contrast to other studies that have shown the beneficial effect of GIPR agonists on adipose metabolism. Previous studies show that how a single amino acid substitution in the GIPR receptor (E354Q) leads to enhanced agonist induced desensitization that impairs the ability of the GIP to control adipose insulin sensitivity (Mohammad et al., 2014). The data from these studies add to the interesting debate whether GIPR activation or GIPR inhibition is the right strategy to treat metabolic abnormalities associated with Type- 2 diabetes.

It is widely documented that GPCR signaling involves crossregulation of many pathways including cross-talks between different GPCRs as well as with other signaling pathways. Besides the acute signaling, GPCR, in direct or through crosstalk, also regulate the development of addictive diseases. In this area (Maccioni et al., 2021), in their paper demonstrate that treatment with non-sedative doses of the novel positiveallosteric modulator (PAM) of the GABA-B receptor, KK-92A ([(4-(cycloheptylamino)-5-(4-(trifluoromethyl)phenyl)pyrimidin2-yl)methanol]) potently and effectively suppressed operant oral alcohol self-administration and cue-induced reinstatement of alcohol-seeking in alcohol-preferring Sp rats. KK-92A has high potency and selectivity for GABA-B. Besides, KK-92A has high bioavailability in the brain and a remarkable in vivo efficacy. The data from this study add to the earlier experimental data on the ability of KK-92A to reduce nicotine self-administration and cueinduced reinstatement of nicotine seeking in rats and therefore, broadening the anti-addictive profile of KK-92A.

Matthees et al. (2021) elucidate the role of GPCR Kinases (GRKs) in the regulation of GPCR signaling. GRKs and $\beta$-arrestins interact with activated GPCRs and regulate their intracellular trafficking. The authors discuss how the expression levels of GRKs, arrestins, and GPCRs play a crucial role in the development of pathological conditions. They analyzed expression data for GRKs and $\beta$-arrestins in 61 tissues annotated in the Human Protein Atlas and presented their analysis in the context of pathophysiological dysregulation of the GPCR/GRK/ $\beta$-arrestin system. This tissue-specific point of view might be the key to unraveling the individual impact of different GRK isoforms on GPCR regulation.

The review by Tian et al. (2020) highlights recent progress regarding the critical components of the JAK2-STAT5 pathway and its crosstalk with G-protein coupled receptor (GPCR) signaling. Hormones are crucial for ductal morphogenesis in the mammary gland. During puberty, estrogen, growth hormone $(\mathrm{GH})$, and prolactin are required for the development of the mammary gland. $\mathrm{GH}$ and prolactin regulate mammary gland function through the phosphorylation of Janus kinase 2 (JAK2) and activation of its downstream regulator signal transducers and activators of transcription 5 (STAT5). The authors evaluate recent data to demonstrate that GPCR activation has a profound impact on the JAK2-STAT5 signaling pathway.

Finally (Li et al., 2021), describe Identification and Functional Analysis of G Protein-Coupled Receptors in steroid hormone, 20Hydroxyecdysone (20E) signaling from the Helicoverpa armigera Genome. The authors show that 20-hydroxyecdysone signals through multiple GPCRs including prolactin-releasing peptide receptor (PRRPR), smoothened (SMO), adipokinetic hormone receptor (AKHR), 5-hydroxytryptamine receptor (HTR), 
Frizzled 7 (FZD7), and tachykinin-like peptides receptor 86C (TKR86C) to regulate growth and development of Helicoverpa armigera.

\section{CONCLUSION}

In conclusion, the research topic contains a fascinating collection of original research papers and review articles encompassing cell biology, physiology, and molecular pharmacology of G-protein coupled receptors. We hope that the data and information conveyed through this research topic will be beneficial to the scientific community in general and researchers in this exciting research area, in particular. We believe the research ideas presented will push more studies to further understand the physiological significance of GPCRs and unearth additional therapeutic possibilities.

\section{REFERENCES}

Alexander, S. P. H., Christopoulos, A., Davenport, A. P., Kelly, E., Mathie, A., Peters, J. A., et al. (2019). The Concise Guide to Pharmacology 2019/20: G Protein-Coupled Receptors. Br. J. Pharmacol. 176, S21-S141. doi:10.1111/bph.14748

Chou, Y.-S., Chuang, S.-C., Chen, C.-H., Ho, M.-L., and Chang, J.-K. (2021). G-Protein-Coupled Estrogen Receptor-1 Positively Regulates the Growth Plate Chondrocyte Proliferation in Female Pubertal Mice. Front. Cel Dev. Biol. 9, 710664. doi:10.3389/fcell.2021.710664

Davenport, A. P., Scully, C. C. G., de Graaf, C., Brown, A. J. H., and Maguire, J. J. (2020). Advances in Therapeutic Peptides Targeting G Protein-Coupled Receptors. Nat. Rev. Drug Discov. 19, 389-413. doi:10.1038/s41573-020-0062-Z

Hanyaloglu, A. C., and Grammatopoulos, D. K. (2017). Pleiotropic GPCR Signaling in Health and Disease. Mol. Cell Endocrinol. 449, 1-2. doi:10.1016/j.mce.2017.05.013

Husted, A. S., Trauelsen, M., Rudenko, O., Hjorth, S. A., and Schwartz, T. W. (2017). GPCR-mediated Signaling of Metabolites. Cel Metab. 25, 777-796. doi:10.1016/j.cmet.2017.03.008

Kizilkaya, H. S., Sørensen, K. V., Kibsgaard, C. J., Gasbjerg, L. S., Hauser, A. S., Sparre-Ulrich, A., et al. (2021). Loss of Function Glucose-dependent Insulinotropic Polypeptide Receptor Variants Are Associated with Alterations in BMI, Bone Strength and Cardiovascular Outcomes. Front. Cel Dev. Biol. 9, 749607. doi:10.3389/fcell.2021.749607

Li, Y.-L., Li, Y.-X., Wang, X.-P., Kang, X.-L., Guo, K.-Q., Dong, D.-J., et al. (2021). Identification and Functional Analysis of G Protein-Coupled Receptors in 20Hydroxyecdysone Signaling from the Helicoverpa Armigera Genome. Front. Cel Dev. Biol. 9, 753787. doi:10.3389/fcell.2021.753787

Lin, Y.-H., Maaroufi, H. O., Kucerova, L., Rouhova, L., Filip, T., and Zurovec, M. (2021). Adenosine Receptor and its Downstream Targets, Mod(mdg4) and Hsp70, Work as a Signaling Pathway Modulating Cytotoxic Damage in Drosophila. Front. Cel Dev. Biol. 9, 651367. doi:10.3389/fcell.2021.651367

Maccioni, P., Kaczanowska, K., Lawrence, H., Yun, S., Bratzu, J., Gessa, G. L., et al. (2021). The Novel Positive Allosteric Modulator of the GABAB Receptor, KK92A, Suppresses Alcohol Self-Administration and Cue-Induced Reinstatement of Alcohol Seeking in Rats. Front. Cel Dev. Biol. 9, 727576. doi:10.3389/ fcell.2021.727576

Mahri, S. A., Ghamdi, A. A., Akiel, M., Aujan, M. A., Mohammad, S., and Aziz, M. A. (2020). Free Fatty Acids Receptors 2 and 3 Control Cell Proliferation by Regulating Cellular Glucose Uptake. Wjgo 12, 514-525. doi:10.4251/WJGO.V12.I5.514

Matthees, E. S. F., Haider, R. S., Hoffmann, C., and Drube, J. (2021). Differential Regulation of GPCRs-Are GRK Expression Levels the Key? Front. Cel Dev. Biol. 9, 687489. doi:10.3389/fcell.2021.687489

Mohammad, S., Patel, R. T., Bruno, J., Panhwar, M. S., Wen, J., and McGraw, T. E. (2014). A Naturally Occurring GIP Receptor Variant Undergoes Enhanced Agonist-Induced Desensitization, Which Impairs GIP Control of Adipose Insulin Sensitivity. Mol. Cell Biol. 34, 3618-3629. doi:10.1128/mcb.00256-14

\section{AUTHOR CONTRIBUTIONS}

All authors listed have made a substantial, direct, and intellectual contribution to the work and approved it for publication. SM, MKG and MB wrote, edited and finalized the paper.

\section{FUNDING}

The work was supported by King Abdullah International Medical Research Center (Grant \# RC20/385 to SM).

\section{ACKNOWLEDGMENTS}

We thank all authors and reviewers for their contribution to the Research Topic.

Mohammad, S. (2015). Role of Free Fatty Acid Receptor 2 (FFAR2) in the Regulation of Metabolic Homeostasis. Cdt 16, 771-775. doi:10.2174/1389450116666150408103557

Morrow, N. M., Hanson, A. A., and Mulvihill, E. E. (2021). Distinct Identity of GLP-1R, GLP-2R, and GIPR Expressing Cells and Signaling Circuits within the Gastrointestinal Tract. Front. Cel Dev. Biol. 9, 703966. doi:10.3389/fcell.2021.703966

Nieto Gutierrez, A., and McDonald, P. H. (2018). GPCRs: Emerging Anti-cancer Drug Targets. Cell Signal. 41, 65-74. doi:10.1016/j.cellsig.2017.09.005

Okuno, Y., Yang, J., Taneishi, K., Yabuuchi, H., and Tsujimoto, G. (2006). GLIDA: GPCR-Ligand Database for Chemical Genomic Drug Discovery. Nucleic Acids Res. 34, D673-D677. doi:10.1093/nar/gkj028

Pavlos, N. J., and Friedman, P. A. (2017). GPCR Signaling and Trafficking: The Long and Short of it. Trends Endocrinol. Metab. 28, 213-226. doi:10.1016/j.tem.2016.10.007

Perez, D. M. (2021). Current Developments on the Role of al-Adrenergic Receptors in Cognition, Cardioprotection, and Metabolism. Front. Cel Dev. Biol. 9, 652152. doi:10.3389/fcell.2021.652152

Pierce, K. L., Premont, R. T., and Lefkowitz, R. J. (2002). Seven-transmembrane Receptors. Nat. Rev. Mol. Cel Biol. 3, 639-650. doi:10.1038/nrm908

Sriram, K., and Insel, P. A. (2018). G Protein-Coupled Receptors as Targets for Approved Drugs: How many Targets and How many Drugs? Mol. Pharmacol. 93, 251-258. doi:10.1124/mol.117.111062

Strange, P. G. (2008). Signaling Mechanisms of GPCR Ligands. Curr. Opin. Drug Discov. Dev. 11, 196-202.

Tian, M., Qi, Y., Zhang, X., Wu, Z., Chen, J., Chen, F., et al. (2020). Regulation of the JAK2-STAT5 Pathway by Signaling Molecules in the Mammary Gland. Front. Cel Dev. Biol. 8, 604896. doi:10.3389/fcell.2020.604896

Wang, J., Gareri, C., and Rockman, H. A. (2018). G-protein-coupled Receptors in Heart Disease. Circ. Res. 123, 716-735. doi:10.1161/CIRCRESAHA.118.311403

Wolf, S., and Grünewald, S. (2015). Sequence, Structure and Ligand Binding Evolution of Rhodopsin-like G Protein-Coupled Receptors: A crystal Structure-Based Phylogenetic Analysis. PLoS One 10, e0123533. doi:10.1371/journal.pone.0123533

Conflict of Interest: The authors declare that the research was conducted in the absence of any commercial or financial relationships that could be construed as a potential conflict of interest.

Publisher's Note: All claims expressed in this article are solely those of the authors and do not necessarily represent those of their affiliated organizations, or those of the publisher, the editors and the reviewers. Any product that may be evaluated in this article, or claim that may be made by its manufacturer, is not guaranteed or endorsed by the publisher.

Copyright (C) 2021 Gupta, Beg and Mohammad. This is an open-access article distributed under the terms of the Creative Commons Attribution License (CC BY). The use, distribution or reproduction in other forums is permitted, provided the original author(s) and the copyright owner(s) are credited and that the original publication in this journal is cited, in accordance with accepted academic practice. No use, distribution or reproduction is permitted which does not comply with these terms. 